\title{
SensApp: a FET-Open project for developing a supersensor able to detect Alzheimer's disease biomarkers in blood
}

\author{
Romina Rega $^{1 *}$, Martina Mugnano ${ }^{1}$, Danila Del Giudice ${ }^{1,2}$, Simona Itri ${ }^{1,2}$, Volodymyr Tkachenko ${ }^{1}$, \\ Veronica Vespini $^{1}$, Sara Coppola ${ }^{1}$, Pietro Ferraro ${ }^{1}$, Heidi Ottevaere ${ }^{3}$, Yunfeng Nie $^{3}$, Sanna Uusitalo ${ }^{4}$, \\ Reinhard Schwoediauer ${ }^{5}$, Martin Kaltenbrunner ${ }^{5}$, Markku Känsäkoski ${ }^{6}$, Emanuela Mazzon ${ }^{7}$, Pier \\ Luca Maffettone $^{8}$, Gaetano D’Avino ${ }^{8}$, Simonetta Grilli ${ }^{1}$ \\ ${ }^{1} \mathrm{CNR}$, Institute of Applied Sciences and Intelligent Systems, Italy \\ ${ }^{2}$ University of Campania "Luigi Vanvitelli”, Viale Abramo Lincoln, 5, 81100, Caserta, Italy \\ ${ }^{3}$ Vrije Universiteit Brussel, Department of Applied Physics and Photonics, Brussels Photonics, Belgium \\ ${ }^{4}$ VTT Technical Research Centre of Finland, Finland \\ ${ }^{5}$ University of Linz, Austria \\ ${ }^{6}$ Ginolis Ltd \\ ${ }^{7}$ IRCCS Centre "Bonino Pulejo", Italy \\ ${ }^{8}$ University of Naples "Federico II", Italy
}

\begin{abstract}
The goal of the SensApp FET-Open project is to develop an innovative super-sensor that will be able to detect Alzheimer's disease (AD) biomarkers ( $\beta$-amyloid, Tau and pTAU) in peripheral blood. Considering that nowadays an accurate diagnosis of AD requires the highly invasive withdrawal and analysis of cerebrospinal fluid, SensApp will represent a breakthrough in the field of $\mathrm{AD}$ diagnosis thanks to the ability to detect the early stage of the disease by a simple blood collection. We call Droplet-Split-and-Stack (DSS) the new technology that will emerge from SensApp. The achievement of SensApp goal is enabled by the interdisciplinary cooperation between different research institutions and one company involved in the key fields of the project, Vrije Universiteit Brussels, VTT Technical Research Centre of Finland, University of Linz, Ginolis Ltd, IRCCS Centre "Bonino Pulejo", under the coordination of CNR-Institute of Applied Sciences and Intelligent Systems. This communication will illustrate the progress of the activities.
\end{abstract}

Keywords: Alzheimer disease; biosensors; SensApp project; Droplet-Spilt-and-Stack.

\section{INTRODUCTION}

Alzheimer's disease (AD) is a serious disease currently very widespread in the world population; it is a degenerative and irreversible neurological disease that leads to death through a state of dementia in the elderly population [1]. It is estimated that around 30 million people are affected by AD today and that number is in continuous increase. The neuropathological hallmarks of $\mathrm{AD}$ are formations of senile plaques composed of $\beta$-Amyloid ( $\beta \mathrm{A})$ ) 1-42 peptides and neurofibrillary tangles (NFTs) consisting of abnormally phosphorylated tau (P-Tau) protein (a microtubule-associated structural protein) in the brain [2]. Senile plaques and NFTs produce several pathogenic mechanisms as well as neurovascular dysfunctions, inflammatory processes, oxidative stress, and mitochondrial dysfunctions, until to degeneration of neurons and synapses [3].

*r.rega@isasi.cnr.it

www.isasi.cnr.it 
Currently, the diagnosis of this pathology takes place through invasive techniques that involve the detection of these specific protein biomarkers (Amyloid-beta, tau, P-tau) in the cerebrospinal fluid (CSF)[4] through immunoassay methods based on ELISA kits (enzyme-linked immunosorbent assay)[5,6], provided by high-value companies such as ThermoFisher, Abcam and Abnova, or through positron emission tomography (PET) of the brain with amyloid tracer[7]. However, these techniques are very expensive and require a specialist intervention and hospitalization of the patient and therefore the disease is commonly diagnosed when it is in an advanced stage. An early diagnosis would make the identification of the biomarkers simple through sampling the peripheral blood where the presence of the biomarkers indicates the presence of the disease. Unfortunately, the current ELISA kits are unable to detect such biomarker levels in peripheral blood due to their abundance well below the standard sensitivity of $50-100 \mathrm{pg} / \mathrm{mL}$. Diffusion phenomena is encountered by antigens and antibodies due to the large reaction volumes $(50 / 100 \mathrm{uL})$ in relatively large areas $(1 / 5 \mathrm{~mm})$, leading to a significantly low signal / noise ratio [8-9].

Numerous non-core approaches have been proposed in the scientific literature to improve this sensitivity. such as: Surface Plasmon Resonance (SPR); biological barcode analysis; Plasmonic ELISA; Digital ELISA; MALDI-TOF mass spectrometry. Most of them have been also tested for the determination of AD-related biomarkers [10]. Although they exhibit a noticeable improvement in sensitivity, they all have limited success for routine clinical practice for the following main reasons. 1) Their common denominator is the use of highly expensive technologies based for example on laser ionization, thin Au coatings, magnetic and Au nanoparticles, or nano-well platforms, accessible only to highly specialized laboratories. 2) They all require time-consuming and highly critical sample preparation that goes far beyond routine practice, thus adding non-negligible uncertainties to the assay and the need for highly qualified personnel. 3) All require sample volumes of at least tens of $\mu \mathrm{L}$, thus preventing applications with very small volumes. All of these drawbacks hinder mass production and accessibility to routine clinical trials.

The SensApp Project received funding from the European Union under the Horizon 2020 Program in 2019 [11] with the aim on the detection of low biomarker levels in clinical blood samples. The objective is to develop, in 3 years, a sensor capable of detecting small molecule traces with concentration below $1 \mathrm{pg} / \mathrm{mL}$ for Alzheimer's disease biomarkers such as b-amyloid, tau protein, phospho-tau protein, indicating a neurological degeneration and typical evolution of $\mathrm{AD}$. This technology could revolutionize the diagnostic technique, since a simple blood sample would help doctors to make a diagnosis and possibly help in the search for a cure.

In order to overcome the ELISA LOD, the SensApp biosensor aims to develop an innovative technology that we call Droplet-Split-and-Stack (DSS) through the use of a pyro-electro-hydrodynamic (p-jet) dispensing system. The DSS is able to accumulate biomarker molecules actively and directly on a micrometer-scale binding site on a solid reaction medium. This allows the antigen-antibody reaction to occur in sub-microliter volumes, avoiding the diffusion limits of standard immunoassays, thus improving the fluorescence signal per unit area and significantly improving the LOD. The project involves the development of a fully automated and cost-effective super-sensor. The project brings together very diverse skills ranging from physics and engineering to biology and clinical sciences. The goal is to implement a prototype that is able to diagnose Alzheimer's quickly and non-invasively. This will radically change doctors' perspectives, as new experimental therapies can be studied in the early stages of the disease, before the irreversible phase, which usually coincides with the appearance of recognizable symptoms such as memory loss. This communication will illustrate the progress of the activities.

\section{P-jet system and DSS approach}

Today the clinical practice for the early diagnosis of the disease, through the determination of antibodies in body fluids, makes use of consolidated immunoassays based on immunological test kits for immunosorbent enzymes (ELISA), chemiluminescence (CL) or (electro) - CL. Their LOD is about $50-100 \mathrm{pg} / \mathrm{ml}$ and is mainly determined by the diffusion efficiency of the involved biomolecules (antigens and antibodies) to find themselves in the detection system [9]. This LOD is extremely crucial when determining antibodies in low concentrations. In order to overcome the ELISA LOD, the project aims to develop an innovative technology (drop -split-and -stack (DSS) that allows to concentrate micro-droplets of the biological sample containing the biomarkers of interest on a very limited area of a support dedicated to chemically bind the biomarker with its antibody.

The underlying principle of the sensor is based on the pyroelectric effect and therefore on the use of ferroelectric crystals such as lithium niobate (NL) and on the manipulation of the fluids under examination in the presence of the pyroelectrically generated electric field (p-jet system). Figure 1 schematically shows the p-jet system. A mother drop (which acts as a reservoir) of the biological sample with little abundant biomarker molecules is loaded inside a loading support which will have a microtechnological orifice that will allow the formation of a thin liquid meniscus on the opposite side. The loading support will be engineered and developed following a study on the fluid dynamic behaviour 
of the biological sample to be treated. A slide, which we call the "reaction support", is functionalized by immobilizing the appropriate antibody (high density and high affinity with the antigen in the sample) and faces the orifice. The pyroelectric crystal, functionalized with a thermal source (appropriately studied) point-wise aligned with the orifice, acts as a source of the pyro-electro-hydrodynamic jet (p-jet) which activates the rapid delivery of tiny droplets ("split") on the same site (" $\mu$-site") as the reaction support. Therefore, after the thermal stimulation (a few degrees with respect to the ambient $\mathrm{T}$ ) there follows an accumulation of uncompensated charge on the crystal due to the pyroelectric effect. According to the pyroelectric effect, this phenomenon is associated with a high electric field that originates from the surface of the crystal [12-14]. This electric field can be used for a wide range of applications from biological to soft matter manipulation [15-19]. In this case, the generated electric field is used for dividing the mother drop (which faces the field) into many small micro-drops that will be accumulated in a single site of micrometre size on the reaction support generating what we call drop-split-and-stack (DSS).

The p-jet aspirates the daughter droplets directly from the meniscus of the mother drop loaded by the operator through a specific orifice, producing the smallest volumes ever seen today from an agile system without nozzle and electrode. Commercial and frontier systems produce droplets with larger or even comparable volumes, but all with the common problem of using a nozzle [20] or through touch-based procedures. This leads them to encounter major problems related to sample channelling, pressure regulation, large dead volumes, cross contamination and clogging [21]. Moreover, the p-jet technology is cheap and could be ready for mass production after this project. The p-jet uses standard procedures for sample preparation, with no additional treatment steps and consequent uncertainties. Recently, p-jet approach was also used for different applications, including, accumulation of other very diluted biomolecule [22,24], the formation of spiral fibres [25] and manipulation of soft matter [26-28].

FIGURE 1 shows the simplified view of the first super-sensor demonstrator. For simplicity, only one DSS channel is shown, but the feasibility of a multi-channel setup will be considered for both high-throughput and multi-target tests.



Figure 1: Simplified view of the p-jet system.

Thanks to this approach, the diffusion problems generally encountered in conventional systems such as ELISA are reduced significantly, since the reaction between the biomarker (antigen) and the respective antibody is forced, maximizing the probability of encounter, thus increasing the fluorescence signal per unit of area. The ambition is to achieve a sensitivity far below the LOD of $50 \mathrm{pg} / \mathrm{mL}$ of the systems currently on the market aiming to achieve a sensitivity below $1 \mathrm{pg} / \mathrm{mL}$. The comparison between the two approaches is schematized in Figure 2 . 



Figure 2: Schematic view of the accumulation effect: a) in standard ELISA deposition: manual drop delivery into a Petri dish used in the ELISA kit; b) In the DSS approach of accumulation with the pjet.

Figure 2 shows schematic view of the accumulation effect in which the target molecules and other antigens (coloured dots) are dispersed in the biological solution. The symbols represented with the $\mathrm{Y}$ indicate the binding molecules present on the reaction slide.

If a solution with a critically low concentration of target molecules (below the LOD of conventional ELISA kits), goes through a reaction producing a fluorescent signal (FL), it is observed that in the approach based on the ELISA kit (FIGURE2 a), after an appropriate incubation period, the molecules to be detected will be diffused in the reaction volume $(100 \mu \mathrm{L})$ until they meet the binding molecules on the surface of the Petri dish. The DSS approach (Figure $2 \mathrm{~b}$ ), on the other hand, allows to dispense the same quantity of molecules on a very limited area of the reaction support $(\sim 1 \div$ $5 \mu \mathrm{m} 2$ ) thus forcing the target molecules to react with the binding ones in a smaller volume.

The difference between these two technologies is that: in the case of the ELISA kit there is a relatively large discrepancy between the volumetric reaction and the concentration of molecules, the effective bonds are distributed over a relatively large surface, thus producing a very weak FL signal per unit area, resulting in an unsatisfactory signal-to-noise ratio (SNR) which makes the detection of molecules inadequate. On the contrary, with the DSS method the FL signal per unit area is much higher, the SNR increases significantly which allows to detect even highly diluted molecules. Therefore, thanks to this approach the reaction will be forced with a high efficiency since the diffusion phenomena will be eliminated, obtaining very strong fluorescence signals. For simplicity, the antigen molecules of Figure 2 are fluorescent but, in the project, are labelled by secondary reactions after accumulation. The antigen-antibody reaction has revolutionary efficacy as follows: (1) the tiny volume of droplets that accumulate will generate a very thin solvent layer with a very low expansion front, thus overcoming the diffusion of antigen molecules; (2) the rapid accumulation of the droplets (about 1 droplet / second) will ensure the continuous hydration of the reaction site, through a very thin layer of solvent in which the antigen molecules are highly accumulated. This thin layer will ensure the successful completion of the immunoreaction where the key (i.e. the antigen) fits into the lock (i.e. the antibody) with a high degree of specificity.

The volume of the droplets depends mainly on the shape of the meniscus of the mother drop and the intensity of the electric field. Therefore, after an in-depth study (accompanied by mathematical simulations) on the fluid dynamic behaviour of the biological fluid to be analysed, in relation to the set-up to be developed and the behaviour in the presence of an electric field, a system suitable for the very high sensitivity detection of biomarkers was developed. 


\section{Research Methodology of the SensApp project}

The SensApp project is strongly guided by experimentation supported by in-depth studies on different aspects: physics of the pyroelectric effect; physics of droplet formation; design and engineering of each module; chemistry of reaction protocols; optical signal FL.

Several research institutes and one company collaborate in the SensApp project and they are involved in the key areas of the project: the Vrije Universiteit Brussels (VUB), Technical Research Centre of Finland (VTT), Linz University (JKU), Ginolis Ltd, IRCCS Center "Bonino Pulejo", with the coordination of the CNR Institute of Applied Sciences and Intelligent systems. The consortium researchers visited their respective laboratories, sharing their know-how in particular on p-jet technology in order to plan and discuss the strategies to be adopted for the microengineering of the building blocks and to integrate them into the first super-sensor demonstrator. The group of the University of Naples Federico II, Department of Materials Engineering (DICMAPI), with experience in fluid physics and computational fluid dynamics, has modelled the behaviour of the different biological fluids to be analysed in the presence of the electric field pyroelectrically generated by a lithium niobate crystal. This study led to the identification of the profile of the meniscus that produces the droplets with the best volume repeatability. Furthermore, on the basis of these studies, the possibility of an appropriate mixing of the biological sample to optimize the surface tension was also evaluated and studied. The result provided the first design input for the orifice geometry produced by the VUB group. The CNR implemented the reaction protocols for each antigen accumulated on the reaction support by means of the p-jet system present in the CNR laboratories. As a first analysis, commercial test samples were used and tested on the bulk p-jet system present in the CNR laboratories. The reaction support is also commercial, and, after accumulation, it is removed from the p-jet system (see Figure 2) and read by a commercial fluorescence scanner. Through meetings and call between the members of the consortium, the procedures for the chemistry of reaction protocols, the integration of the blocks were designed and implemented to then proceed with mechanical design, integration and assembly regarding the three layers.

\subsection{Chemistry of reaction protocols (CNR- IRCCS Center "Bonino Pulejo")}

A reliable immunoreaction protocol compatible with the droplets deposited by p-jet on the reaction support was implemented according to the design concept of the super-sensor prototype. A pure solution of $\beta$ Amyloid protein fragment 1-40 (A $\beta 1-40)$ and of $\beta$ Amyloid protein fragment 1-42 (A $\beta 1-42)$ were used as model samples. These have as precursor the Amyloid Precursor Protein (APP). For brevity, we call here protein solution (PS) the solution of A $\beta 1-40$ dissolved in $\mathrm{dH}_{2} \mathrm{O}$, and protein solution (PS2) the solution of $\mathrm{A} \beta 1-42$ dissolved in an appropriate buffer. Eventual additional mixing buffers are mentioned specifically for each test. Figure 3 shows the schematic view of the protocol steps composed of 6 phases: Deposition, incubation, blocking of the solution molecules, primary antibody reaction, secondary antibody reaction (which is conjugated with a fluorophore) and reading of the fluorescence signal through the commercial scanner. It was tested first for the $A \beta 1-40$ and successively for the $A \beta 1-42$.

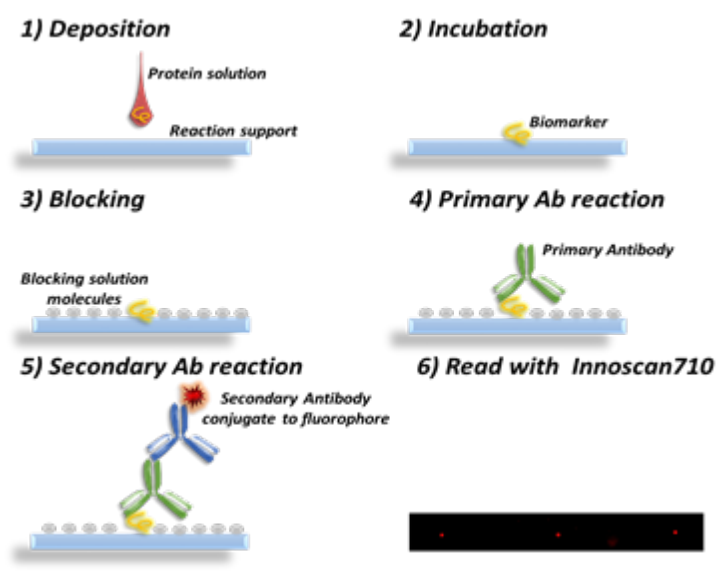

Figure 3. Schematic view of the typical protocol steps. 


\subsection{Testing of the three individual layers}

\subsubsection{The loading support (VUB, CNR)}

The microengineered loading supports were designed and fabricated by VUB and then delivered to CNR. The aim of these tests was to find the orifice configuration that produces a sequence of tiny droplets with a repeatable volume, in order to accumulate as much as possible, the protein molecules and to enhance the fluorescence signal. Different manufacturing conditions have been tested in terms of diameter and wall inclination.

The dispensing has proven to be highly repeatable with spot diameter around $100 \mu \mathrm{m}$. Figure 4 shows the typical spots obtained by p-jet in case of a fluorescent probe.

\section{a)}

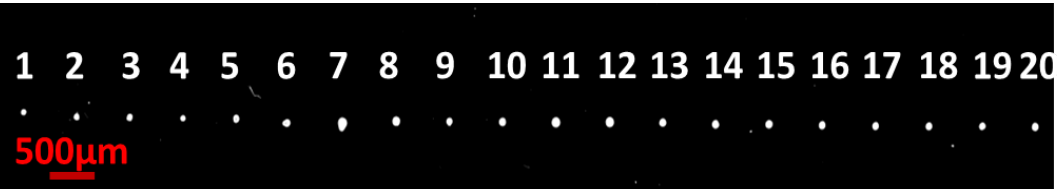

b)

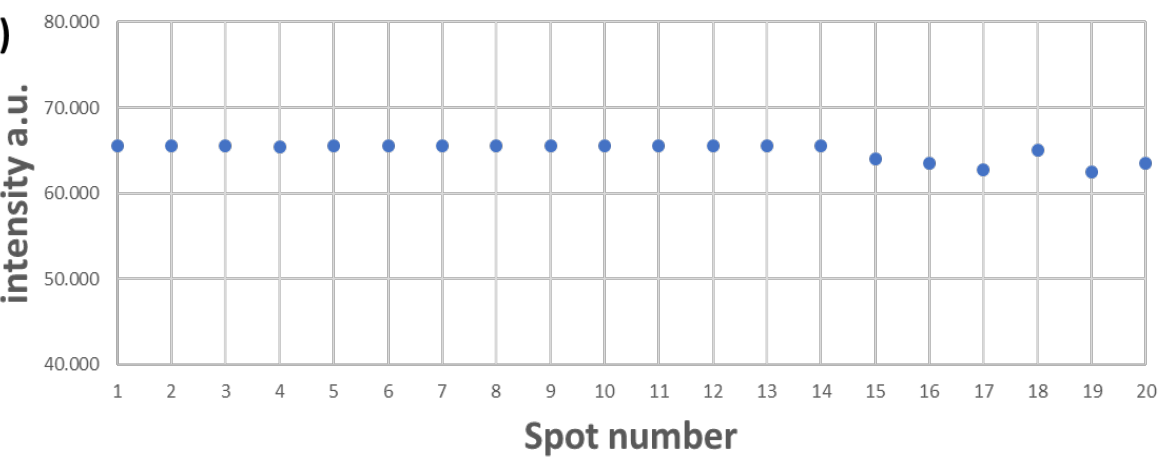

Figure 4: Twenty replicates of spots generated by p-jet with a fluorescent probe (Alexa Fluor).

\subsubsection{The pyroelectric/reaction layer (JKU, CNR)}

The conditions of greater electric field efficiency were analyzed in relation to the thickness and geometry of the crystal to be used, complementing these studies with mathematical simulations. JKU explored the potential of using different materials for creating the pyroelectric effect, such as pyroelectric crystals, ceramics and polymer films. The work started with a focus on thin foils of poled poly (vinylidene fluoride) (PVDF). A few PVDF foils with different thicknesses were experimentally characterized. The laser induced modulation method (LIMM) was applied to determine the pyroelectric coefficient. A second experiment was designed to study the general dynamics of the external electric field, which is produced by a pyroelectric thin film element during a temperature change. The experiment also allowed a rudimentary investigation of the electro-hydrodynamic field effect on silicon oil and thus showed the related possibility of p-jetting events. A specially designed, LASER machined micro-heater enabled repeatable conditions for the experiment. The experiments with PVDF revealed a strong unfavorable charge trapping effect. This effect makes the material less suitable for the intended application as a source for an electric field of sufficient strength and energy. Similar experiments with small crystal plates of $\mathrm{LiNbO} 3$ produced different and much better results. Detailed surface potential measurements of heated LiNbO3 crystal plates were performed under various conditions. The results clearly show a more reduced charge trapping effect for $\mathrm{LiNbO} 3$ compared to PVDF. A theoretical analysis was performed with detailed calculations in order to explain the experimental data. Also, different reaction layers and their influence on the electric field were studied. It was found that, depending on the material, the reaction layer can significantly affect the electrodynamics and screen the electric field from the pyroelectric element.

Different sources of thermal stimulation were also tested through integrated devices (eg copper film micro-heaters, Peltier systems) in order to induce heating on the material while preserving the biological nature of the biomarker to be detected. All the electrothermal properties of the devices were first modeled and then experimentally tested. Great attention was paid to temperature gradients (including negative ones) to avoid harmful effects on biomarker molecules. Each part of the system created was iteratively tested at the CNR using the p-jet system. Finally, a tungsten wire-based heater was selected for the developed pyroelectric layer demonstrator. 


\subsubsection{The optical detection layer (VUB, VTT)}

The general principle of the detection layer is to excite the fluorescence-labelled protein by using a suitable light source, then collect the desired fluorescence (FL) signals and reject the unwanted light as much as possible for a super-sensitive detection of extremely low concentration solutions. The complete light path is illustrated in Figure 6, consisting of the light source, the beam shaper, the reaction slide, the collection optics, the optical filter and the detector. There are many possibilities for each component with emphasis on different capabilities. Therefore, VUB and VTT have characterized the specifications of the crucial components before the design of the final optical detection layer.

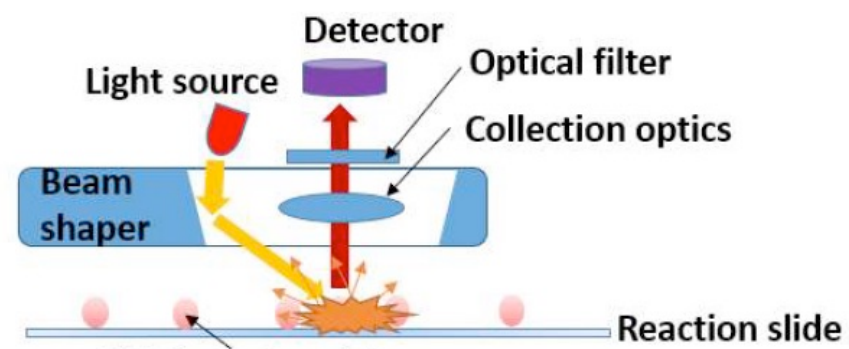

FL labelled protein

Figure 6: The overall light path of the optical detection layer

A preliminary test set-up was built to distinguish the important design parameters for the signal read-out. A Labview program was developed to control all the electronic components, including the XY scanning, the laser diode driver, the photomultiplier (PMT) plus the data acquisition device (DAQ). The test samples were prepared by dissolving Alexa Fluor 647 Carboxylic Acid (Thermo Fisher A33084) into PBS solution. $1 \mu 1$ samples were prepared onto a clean microscope cover slide (both from Thermo and Thorlabs, \#1.5), and waited to dry under room temperature and humidity. By adopting the lessons learnt from the theoretical design as well as the practical experiments, the layout of the detection layer as shown in Figure 7 was built.

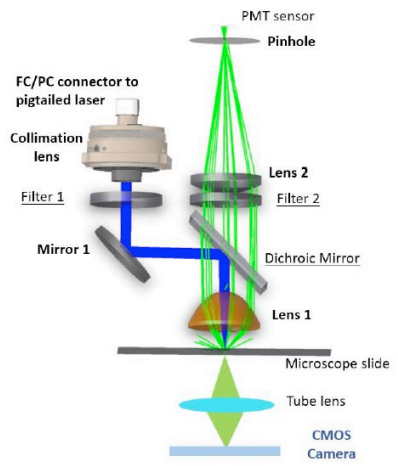

Figure 7: The optical layout of the final detection layer

A camera system was added under the sample slide to enable precise device calibration and to find the sample spots during development phase by using a blue LED illumination. Currently, the Amine slide is seen as the best option for preparing the sample, due to high optical transparency.

\subsection{Integration and assembly of three layers}

\subsubsection{Mechanical design of the loading support and pair pyroelectric layer (VTT, CNR)}

The DSS dispenser unit integrates the loading layer, reaction layer and pyroelectric layer, respectively introduced in Sec. 1.2.1 and 1.2.2. The design transforms the test set-up into a compact prototype with automated translation functions. The system incorporates imaging the spot dispensing with a side view imaging system, automated sample slide translation, automated contact between pyro-electric layer and reaction layer, and operator-controlled loading layer adjustment and spot dispense control. In addition, the system transfers information of the spot locations from to the readout unit and images the spot dispense quality with a top-view down imaging system. The spot locations are determined from one reaction layer corner and the readout unit will have pre-information for the signal read-out locations. The reaction layer 
holders of the DSS dispensing unit and readout unit are identical and have a requirement for the reaction layer to be inserted into the holder in same orientation. Figure 8(a) shows the DSS dispensing unit design that includes the orifice and the pyroelectric layer. Figure 8(b) shows a closeup image of the integration of the loading support and the pair pyroelectric layer with side-view imaging.

(a)

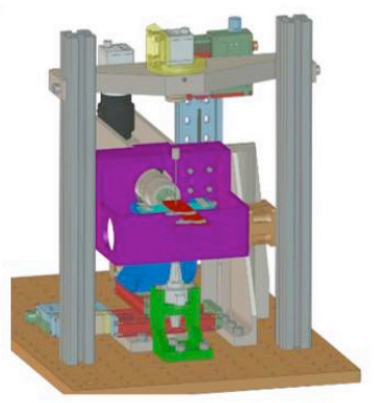

(b)

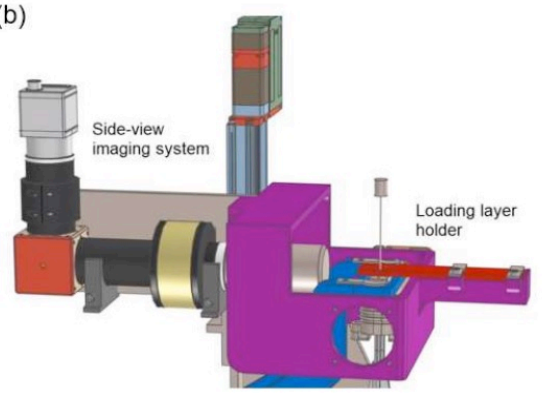

Figure 8: The DSS dispensing unit integrating the loading layer, pyro-electric layer and reaction layer and (b) Close-up of the integration of the loading support and the pair pyroelectric layer

The cooling of the system is insured using metal base for pyroelectric stage. It incorporates the Tungsten wire heating system with pulsed heater cycles. In addition, the system includes a fan blowing air to the cooling structures of pyroelectric stage, $\mathrm{LiNbO} 3$ crystal and reaction layer. The aim of the efficient cooling is to enable faster heating cycles for the DSS dispensing. The system translates the reaction layer in X-Y to enable dispensing the sample spots in a matrix configuration using two $50 \mathrm{~mm}$ Zaber translation stages. In addition, the system translates the loading layer in Zdirection with a Zaber $100 \mathrm{~mm}$ translation stage. Thus, the tip of the loading layer needle can be adjusted to optimal distance from reaction layer upper surface for the dispensing.

\subsubsection{Mechanical design of the integrated detection layer (VTT, VUB)}

The readout unit integrates the main components of the detection layer. It includes the optical layout, automated sample alignment, sensor to ensure optimal distance of the sample slide upper surface from the detection optics, automated shutters, imaging camera for system alignment and a place for the photodiode for laser stability control during the system pre-testing. Figure 9 shows the implementation of the optical layout and the mechanical design for the readout detector.
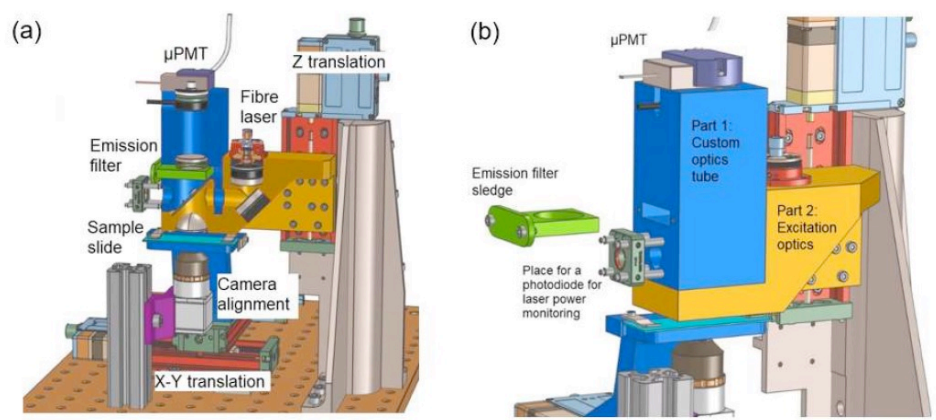

Figure 9: (a) The integration of the optical layout into a readout unit and (b) Image showing the custom opto-mechanics for the readout unit.

The sensitivity of the detection of fluorophore signals sets requirements for the tuning of the readout system as well as the position control of the $\mathrm{X}-\mathrm{Y}-\mathrm{Z}$ directions. The imaging system will be used in the tuning of the optical paths to ensure that the excitation and signal collection overlap with high precision. A photodiode will be used to determine a sufficient stabilization time for the fiber laser after switching on. There is a period, where the temperature of the holder affects the laser stability. This will be taken into account in the measurement process. In addition, there is an automated shutter in front of the excitation laser ensuring the photo bleaching of the fluorescence can be avoided during sample slide positioning without the need for turning the laser off. Another shutter is positioned in front of the $\mu$ PMT detector to 
prevent the accumulation of electric charges to the $\mu$ PMT active material during the sample slide insertion due to ambient illumination.

\section{CONCLUSION}

Many activities related to the project have been delayed due to the COVID-19 emergency, therefore, the European community has granted the project 6 months extension. In conclusion, this project aims to develop a simple and inexpensive test for identifying a panel of biomarkers related to $\mathrm{AD}$ disease directly from a drop of blood where the concentration of the indicative biomolecules of the disease is very low, thus allowing for an early diagnosis. After the SensApp project, DSS has a realistic perspective for easy miniaturization into a portable system for point-of-care applications. All these advances will allow the DSS to exceed the limits of conventional techniques towards an easy determination of low abundance biomarkers in the blood and to allow an early diagnosis to be reached through a routine clinical test (even by capillary puncture). This paves the way for the first time for a variety of advances in diagnosing AD. An enormous impact on society is expected for both the quality of life and the financial balance.

\section{ACKNOWLEDGMENTS:}

The authors acknowledge the EU funding within the Horizon 2020 Program, under the FET-OPEN Project "SensApp", Grant Agreement n.829104.

\section{REFERENCES}

[1] Https://www.who.int/news-room/fact-sheets/detail/dementia. https1.pdf.

[2] Galozzi, S., Marcus, K. \& Barkovits, K. “Amyloid- $\beta$ as a biomarker for Alzheimer's disease: Quantification methods in body fluids". Expert Rev. Proteomics 12, 343-354 (2015).

[3] Blennow, K., de Leon, M. J. \& Zetterberg, H. Alzheimer's disease. Lancet 368, 387-403 (2006).

[4] Kaj Blennowa, Bruno Duboisb, Anne M. Faganc, Piotr Lewczukd, Mony J. de Leone, f, and H. H. "Clinical utility of cerebrospinal fluid biomarkers in the diagnosis of early Alzheimer's disease". Alzheimers Dement. 11, 58-69 (2015).

[5] Jensen, M. et al. "Quantification of Alzheimer amyloid beta peptides ending at residues 40 and 42 by novel ELISA systems”. Mol. Med. 6, 291-302 (2000).

[6] Hansson, O. et al. "Evaluation of plasma $A \beta 40$ and $A \beta 42$ as predictors of conversion to Alzheimer's disease in patients with mild cognitive impairment". Neurobiol. Aging 31, 357-367 (2010).

[7] Aschenbrenner, A. J., Gordon, B. A., Benzinger, T. L., Morris, J. C., \& Hassenstab, J. J. "Influence of tau PET, amyloid PET, and hippocampal volume on cognition in Alzheimer disease". Neurology, 91(9), e859-e866, (2018).

[8] Shena, M. (ed. Biotechniques Books: Microarray Biochip Technology, 1st edn (Eaton Publishing Company, 2000)

[9] Agnello, L., Piccoli, T., Vidali, M., Cuffaro, L., Lo Sasso, B., Iacolino, G., ... \& Ciaccio, M. "Diagnostic accuracy of cerebrospinal fluid biomarkers measured by chemiluminescent enzyme immunoassay for Alzheimer disease diagnosis." Scandinavian journal of clinical and laboratory investigation, 80(4), 313-317, (2020).

[10] Nakamura, A. et al. "High performance plasma amyloid- $\beta$ biomarkers for Alzheimer's disease." Nature 554, 249 254 (2018).

[11] SensApp. SensApp http://www.sensapp.eu/.

[12] Bhowmick, S., Iodice, M., Gioffrè, M., Breglio, G., Irace, A., Riccio, M., ... \& Coppola, S. "Investigation of pyroelectric fields generated by lithium niobate crystals through integrated microheaters." Sensors and Actuators A: Physical, 261, 140-150, (2017).

[13] Rega, R., Gennari, O., Mecozzi, L., Grilli, S., Pagliarulo, V., \& Ferraro, P. "Bipolar patterning of polymer membranes by pyroelectrification." Advanced Materials, 28(3), 454-459, (2016).

[14] Rega, R., Gennari, O., Mecozzia, L., Grilli, S., Pagliarulo, V., \& Ferraro, P. "Pyro-electrification of polymer membranes for cell patterning," In AIP Conference Proceedings (Vol. 1736, No. 1, p. 020042). AIP Publishing LLC, (2016).

[15] Pagliarulo, V., Gennari, O., Rega, R., Mecozzi, L., Grilli, S., \& Ferraro, P. "Twice electric field poling for engineering multiperiodic Hex-PPLN microstructures." Optics and Lasers in Engineering, 104, 48-52, (2018). 
[16] Gennari, O., Marchesano, V., Rega, R., Mecozzi, L., Nazzaro, F., Fratianni, F., ... \& Ferraro, P. "Pyroelectric effect enables simple and rapid evaluation of biofilm formation." ACS applied materials \& interfaces, 10(18), 1546715476, (2018).

[17] Gennari, O., Rega, R., Mugnano, M., Oleandro, E., Mecozzi, L., Pagliarulo, V., ... \& Ferraro, P. “A skin-over-liquid platform with compliant microbumps actuated by pyro-EHD pressure." NPG Asia Materials, 11(1), 1-8, (2019).

[18] Rega, R., Gennari, O., Mecozzi, L., Pagliarulo, V., Mugnano, M., Oleandro, E., ... \& Grilli, S. "Pyro-electrification of freestanding polymer sheets: a new tool for cation-free manipulation of cell adhesion in vitro." Frontiers in chemistry, 7, (2019).

[19] Lettieri, S., Rega, R., Pallotti, D. K., Gennari, O., Mecozzi, L., Maddalena, P., ... \& Grilli, S. "Direct evidence of polar ordering and investigation on cytophilic properties of pyroelectrified polymer films by optical second harmonic generation analysis." Macromolecules, 50(19), 7666-7671, (2017).

[20]Croom, B. P., Abbott, A., Kemp, J. W., Rueschhoff, L., Smieska, L., Woll, A., ... \& Koerner, H. "Mechanics of nozzle clogging during direct ink writing of fiber-reinforced composites." Additive Manufacturing, 37, 101701, (2021).

[21] Du, Z., Yu, X., \& Han, Y. "Inkjet printing of viscoelastic polymer inks.” Chinese Chemical Letters, 29(3), 399-404, (2018).

[22] Rega, R., Martinez, J. M., Mugnano, M., Oleandro, E., Gennari, O., Orlando, P., ... \& Grilli, S. “A pyroelectricbased system for sensing low abundant lactose molecules". In Optical Methods for Inspection, Characterization, and Imaging of Biomaterials IV International Society for Optics and Photonics. Vol. 11060, p. 1106009, (2019).

[23] Rega, R., Mugnano, M., del Giudice, D., Itri, S., Tkachenko, V., Vespini, V., ... \& Grilli, S. "Highly sensitive detection of low abundant molecules by pyro-electrohydro-dynamic jetting". In Biophotonics in Point-ofCare International Society for Optics and Photonics Vol. 11361, p. 113610N, (2020)

[24] Rega, R., Mugnano, M., Oleandro, E., Tkachenko, V., Del Giudice, D., Bagnato, G., ... \& Gangemi, S. "Detecting Collagen Molecules at Picogram Level through Electric Field-Induced Accumulation." Sensors, 20(12), 3567, (2020).

[25] Mecozzi, L., Gennari, O., Rega, R., Grilli, S., Bhowmick, S., Gioffrè, M. A., ... \& Ferraro, P. "Spiral formation at the microscale by $\mu$-pyro-electrospinning". Soft matter, 12(25), 5542-5550, (2016).

[26] Mecozzi, L., Gennari, O., Coppola, S., Olivieri, F., Rega, R., Mandracchia, B., ... \& Grilli, S. "Easy Printing of High Viscous Microdots by Spontaneous Breakup of Thin Fibers". ACS applied materials \& interfaces, 10(2), 2122-2129, (2018).

[27] Rega, R., Gennari, O., Mecozzi, L., Pagliarulo, V., Bramanti, A., Ferraro, P., \& Grilli, S. "Maskless arrayed nanofiber mats by bipolar pyroelectrospinning". ACS applied materials \& interfaces, 11(3), 3382-3387, (2019).

[28] Mecozzi, L., Gennari, O., Rega, R., Battista, L., Ferraro, P., \& Grilli, S. "Simple and rapid bioink jet printing for multiscale cell adhesion islands". Macromolecular bioscience, 17(3), 1600307, (2017). 